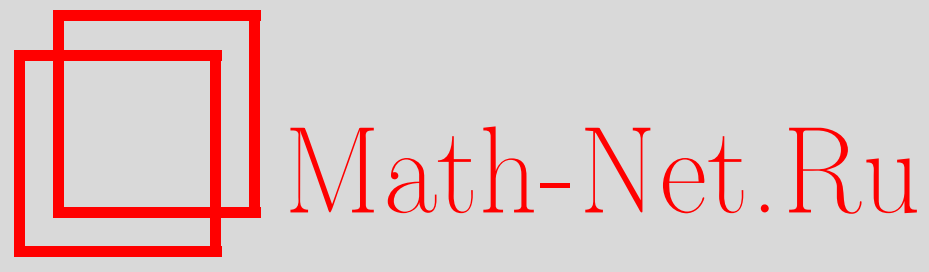

В. П. Маслов, Термодинамика наноструктур, Матем. заметки, 2008, том 84, выпуск 4, 634-637

DOI: https://doi.org/10.4213/mzm6141

Использование Общероссийского математического портала Math-Net.Ru подразумевает, что вы прочитали и согласны с пользовательским соглашением http://www . mathnet.ru/rus/agreement

Параметры загрузки:

IP : 54.197 .130 .99

26 апреля 2023 г., 15:16:43 


\section{Термодинамика наноструктур}

\section{В. П. Маслов}

В статистической физике, т.е. в задаче многих частиц, полагается обычно, что потенциал взаимодействия между двумя частицами априори задан. Ничего подобного в ядерной физике, квантовой теории поля, как правило, нет.

В настоящей работе, как и в предыдущих работах, мы постараемся показать, что в статистической физике такая модель, при которой потенциал взаимодействия зависит только от координат, очень ограничена (см., например, работу автора об исключении фононов [1]).

Для фрактальной размерности $2<d<3$ (т.е. для нанопористой среды) мы будем рассматривать взаимодействие частиц вида потенциала Леннарда-Джонса

$$
\Phi(r)=4 \varepsilon\left\{\left(\frac{a}{r}\right)^{12}-\left(\frac{a}{r}\right)^{6}\right\}
$$

где $\varepsilon$ и $a$ - постоянные, имеющие размерность энергии и длины соответственно; $r^{2}=$ $\left(x_{i}-x_{j}\right)^{2}+\left(y_{i}-y_{j}\right)^{2}+\left(z_{i}-z_{j}\right)^{2}, i \neq j, x_{i}, y_{i}, z_{i}$ - координаты $i$-й частицы. Длина $a$ есть эффективный поперечник частицы, при $r=a$ имеем $\Phi(a)=0$. Величина $\varepsilon$ определяет глубину потенциальной ямы $\Phi(r)$ и, следовательно, характеризует интенсивность межмолекулярных сил.

Обычное уравнение для одетого потенциала [2] в силу [3] должно иметь вид

$$
u(x)=4 \pi^{2} \frac{\int_{\xi \in V_{d}} \Phi(|x-\xi|)\left\{\int_{0}^{\infty} \frac{p^{d-1} d p}{e^{b\left(p^{2} / 2 m+u(\xi)\right)}-1}\right\}|\xi|^{d-1} d|\xi|}{\int_{\xi \in V_{d}}\left\{\int_{0}^{\infty} \frac{p^{d-1} d p}{e^{b\left(p^{2} / 2 m+u(\xi)\right)}-1}\right\}|\xi|^{d-1} d|\xi|} .
$$

Здесь $\Phi(x-\xi)$ - потенциал $(1), \xi \in V_{d}$, а $V_{d}$ - объем:

$$
V_{d}=4 \pi^{2} \int_{0}^{R} r^{d-1} d r=\frac{4}{d} \pi^{2} R^{d}, \quad 2<d \leqslant 3
$$

(см. [3]).

Мы будем полагать, что $x$ лежит в объемчике радиуса порядка $\left(V_{d}^{\text {уд }}\right)^{1 / 3}$ (удельного), т.е. $V_{d}^{\text {уд }}=V_{d} / N$, где $N$ - число частиц.

Но интеграл (2) расходится при $|x-\xi|=0$ как $1 /|x-\xi|^{4}$. Поэтому как правило, полагают, что потенциал Ленарда-Джонса ограничен при $r<r_{0}$, а значит, существенно зависит от $r_{0}$ и стремится к бесконечности при $r_{0} \rightarrow 0^{1}$. Мы перейдем к зависимости от другого параметра, так называемого прицельного параметра $\rho$, к пучку внутри объемчика $V_{d}^{\text {уд }}$, предполагая [4], что пучок однороден по всему своему сечению. Это позволит нам проинтегрировать полученное ниже распределение по эффективному сечению $d \sigma=4 \pi \rho d \rho$. Заметим, что, как распределено $r_{0}$, неизвестно.

Применение результатов классической задачи рассеяния к пористой среде [5], в особенности к нанопористой среде может вызвать непонимание со стороны физиков и математиков, недостаточно хорошо знакомых с соотношениями между теми параметрами, которые там возникают (см. [6], [7]). Однако для того, чтобы это строго обосновать, нужен достаточно большой объем текста.

Работа выполнена при поддержке Российского фонда фундаментальных исследований (грант № 08-01-00601-a).

${ }^{1}$ Обычная математическая регуляризация теории обобщенных функций здесь не подходит. 
Поскольку $x \in V_{d}^{\text {уд }}$, находящемуся вдали от границы объема $V_{d}$, то сделав замену $|\xi-x|=s$ и переходя к пределу при $R \rightarrow \infty$, в силу того, что $\Phi(r)$ быстро убывает, мы получим интеграл по $s$ по всему пространству, а следовательно, $u(x)$ не будет зависеть от $x$, а будет зависеть только $r_{0}$. Именно

$$
u(x)=\frac{16 \pi}{d} \varepsilon a^{d}\left\{\frac{1}{12-d}\left(\frac{a}{r}\right)^{12-d}-\frac{1}{9-d}\left(\frac{a}{r}\right)^{9-d}\right\}
$$

при условии, что $r_{0}$ не зависит от $p$. Но при учете задачи рассеяния $r_{0}$ определяется из соотношения [4]

$$
\frac{\rho^{2}}{r_{0}^{2}}+\frac{m \Phi\left(r_{0}\right)}{\left(p-p^{\prime}\right)^{2}}=1
$$

где $p$ и $p^{\prime}-$ импульсы соответственно частиц, отвечающих координатам $x$ и $\xi$.

Вероятность нахождения частицы с координатой $\xi$ непостоянна на интервале от $r_{0}$ до бесконечности. Она очевидно пропорциональна времени пребывания частицы в интервале $\left(\xi^{\prime}, \xi^{\prime \prime}\right)$, а это время обратно пропорционально скорости частицы $\xi$ относительно частицы $x$. Нетрудно убедиться в том, что эта вероятность равна

$$
F(\xi)=\frac{\left|\left[\frac{1}{m}\left(p_{1}-p_{2}\right)^{2}-\frac{\rho\left(p_{2}-p_{1}\right)^{2}}{m(x-\xi)^{2}}-\Phi(x-\xi)\right]^{-1 / 2}-\left(\frac{1}{m}\left(p_{1}-p_{2}\right)^{2}\right)^{-1 / 2}\right|}{\int_{r_{0}}^{\infty}\left|\left[\frac{1}{m}\left(p_{1}-p_{2}\right)^{2}-\frac{\rho\left(p_{2}-p_{1}\right)^{2}}{m(x-\xi)^{2}}-\Phi(x-\xi)\right]^{-1 / 2}-\left(\frac{1}{m}\left(p_{1}-p_{2}\right)^{2}\right)^{-1 / 2}\right| d r}
$$

Отметим, что перед интегралом стоит малый параметр $b$. Чтобы показатель экспоненты не был отрицательным, необходимо добавить химпотенциал $\mu(\rho)$, который определяется из условия

$$
u(0, \rho)=b|\mu(\rho)| .
$$

Поскольку $x \in V_{d}^{\text {уд }}$ и находится далеко от границы объема, мы получим следующий вид интегрального уравнения. Это интегральное уравнение определяет одетый потенциал

$$
\begin{aligned}
u(p, \rho)=2 \pi \int_{0}^{\infty} & \int_{0}^{2 \pi} \frac{\int_{r_{0}}^{\infty} \Phi(r)\left[\left\{\frac{\left(p-p^{\prime}\right)^{2}}{m}-\frac{\left(p-p^{\prime}\right)^{2} \rho^{2}}{m r^{2}}-\Phi(r)\right\}^{-1 / 2}\right] r^{d-1} d r}{\int_{r_{0}}^{\infty}\left|\left[\left\{\frac{\left(p-p^{\prime}\right)^{2}}{m}-\frac{\left(p-p^{\prime}\right)^{2} \rho^{2}}{m r^{2}}-\Phi(r)\right\}^{-1 / 2}-\frac{\sqrt{m}}{\left|p-p^{\prime}\right|}\right]\right| d r} \\
& \times \frac{1}{e^{b\left(\left(p^{\prime}\right)^{2} / 2 m+u\left(p^{\prime}, \rho\right)\right)-\mu b}-1}\left|p^{\prime}\right|^{d-1} d\left|p^{\prime}\right| d \phi \\
& \times\left\{\int_{0}^{\infty} \int_{0}^{2 \pi} \frac{1}{e^{b\left(p^{2} / 2 m+u(p, \rho)\right)-\mu b}-1} p^{d-1} d p d \phi\right\}^{-1} .
\end{aligned}
$$

Здесь $b=\varsigma / R^{2} k T$ (о константе $\varsigma$ см. [3]), $\mu \leqslant 0$, а $\phi$ - угол на плоскости рассеяния. Теперь мы можем, учитывая, что $\rho$ меняется до $\left(V_{d}^{\text {уд }}\right)^{1 / 3}$, проинтегрировать распределение

$$
\frac{1}{\left(V_{d}^{\mathrm{y} \mu}\right)^{2 / 3}} \int_{0}^{\left(V_{d}^{\mathrm{y} \mu}\right)^{1 / 3}} \frac{1}{e^{b\left(p^{2} / 2 m+u(p, \rho)-\mu\right)}-1}\left\{\int_{0}^{\infty} \int_{0}^{\pi} \frac{1}{e^{b\left(p^{2} / 2 m+u(p, \rho)-\mu\right)}-1} p^{d-1} d p d \phi\right\}^{-1} \rho d \rho
$$

по эффективному сечению

$$
d \sigma=\rho d \rho,
$$

и получить окончательную формулу для распределения типа Бозе-Эйнштейна [8], [9], учитывающее взаимодействие. 
ЗАмечАние 1. Поскольку выражение

$$
\frac{F\left(x_{2}\right)}{e^{b\left(p_{2}^{2} / 2 m+u\left(p_{2}, \rho\right)\right)-b \mu}-1}\left[\iint_{0}^{2 \pi}\left(e^{b\left(p_{2}^{2} / 2 m+u(p, \rho)\right)-b \mu}-1\right)^{-1}\left|p_{2}\right|^{d-1} d p_{2} d \phi\right]^{-1}
$$

проинтегрированное сначала по $x_{2}$, а затем по $d p$, равно единице, то вероятности независимы, и распределение по $x_{2}$ и по $p_{2}$ равно произведению распределений по $x_{2}$ и по $p_{2}$. Нетрудно убедиться, что отсюда будет следовать (7).

ЗАмечАние 2. Как автор уже неоднократно отмечал, распределение Гиббса пишут в виде $e^{-H(p, q) / k T}$, т.е. по существу без малого параметра в экспоненте. Но поскольку интеграл по $p, q$ от этого выражения равен числу частиц $N$, а $N \rightarrow \infty$, то малый параметр в экспоненте необходим. Поэтому если его не вводить, как это делал автор, то нужно полагать $k T \rightarrow \infty$, и малые температуры не рассматривать вообще или же считать, что константа Больцмана $k \rightarrow \infty$. Но это слишком непривычно (в отличие от случая, когда принято считать, что константа Планка стремится к нулю). Соответственно этому нужно $b$ в потенциале Ленарда-Джонса $\varepsilon \rightarrow \infty$, или же делить его на безразмерный параметр $b k T$, что мы и сделаем. Тогда (5) превратится в

$$
\frac{\rho^{2}}{r_{0}^{2}}+\frac{\frac{m}{b k T} \Phi\left(r_{0}\right)}{\left(p-p^{\prime}\right)^{2}}=1
$$

поэтому в предыдущих формулах мы должны заменить взаимодействие Леннарда-Джонса $\Phi$ на $\Phi / b k T$, которое можно вновь обозначить через $\Phi$.

Фактор сжимаемости $Z$ равен

$$
\begin{aligned}
Z=\frac{b}{\left(V_{d}^{\mathrm{y}}\right)^{2 / 3}} \int_{0}^{V_{\text {уд }}^{1 / 3}}\left[\int_{0}^{\infty} \int_{0}^{2 \pi} \frac{\frac{p^{2}}{2 m} d p^{3} d \phi}{e^{b\left(p^{2} / 2 m+u(p, \rho)\right)-b \mu}-1} /\right. \\
\left.\qquad \int_{0}^{\infty} \int_{0}^{2 \pi} \frac{d p^{3} d \phi}{e^{b\left(p^{2} / 2 m+u(p, \rho)\right)-b \mu}-1}\right] \rho d \rho .
\end{aligned}
$$

Если температура задана (изотермический процесс) и задан химпотенциал, зависящий от нее, тогда $P-V_{\text {уд }}$ диаграмма, т.е. зависимость давления от объема, выражается в силу вышеизложенного следующим образом

$$
P=\frac{k T Z}{V_{d}^{\mathrm{y}}},
$$

где $u(p, \rho)$ удовлетворяет интегральному нелинейному уравнению (7). Здесь дается модификация определения давления, согласованная со случаем идеального газа.

Уравнение (10) является аналогом уравнения Ван-дер-Ваальса и других эмпирических соотношений. Оно определяет фазовые переходы.

Мы, таким образом, приходим к модификации термодинамических соотношений при учете взаимодейстия для наноструктур. Сделав замену $b p^{2}=\bar{p}^{2}$ и $b p^{\prime 2}=\bar{p} / 2$ мы избавляемся во всех соотношениях от малого параметра $b$ и приходим к окончательным формулам. Эта же замена позволяет исключить малый параметр и в интегральном уравнении для $u(p, \rho)$. При этом написанные новые соотношения будут соответствовать рассмотрению физиков, которые игнорируют малый параметр $a^{d-1} / V_{d}^{2 / 3}$, что и разумно, потому что в окончательных ответах он пропадает. Однако для строгих доказательств он необходим.

В заключение отметим, что для того чтобы найти критические константы (температуру и т.д.), в силу вышеприведенных формул, достаточно исследовать критические точки решения интегрального уравнеия $u(p, \rho)$ при $\rho=\left(V_{d}^{\text {уд }}\right)^{1 / 3}$. 


\section{СПИСОК ЦИТИРОВАННОЙ ЛИТЕРАТУРЫ}

[1] V.P. Maslov, Russ. J. Math. Phys., 6:4 (1999), 409-425. [2] В.П. Маслов, Мaтем. заметки, 83:6 (2008), 864-879. [3] V. P. Maslov, Math. Notes, 84:3 (2008), 446-448. [4] Л. Д. Ландау, Е. М. Лифшиц, Теоретическая физика, т. 5: Статистическая физика, Наука, М., 1964. [5] В.П. Маслов, В.П. Мясников, В.Г. Данилов, Математическое моделирование аварийного блока Чернобыльской АЭС, Наука, М., 1987. [6] В. П. Маслов, Квантование термодинамики и ультравторичное квантование, Ин-т компьютер. исслед., М., 2001. [7] В.П. Маслов, И. А. Молотков, Теплофизика высоких температур, 2008 (в печати). [8] В.П. Маслов, В. Е. Назайкинский, Матем. заметки, 83:6 (2008), 880-898. [9] В. П. Маслов, В. Е. Назайкинский, Матем. заметки, 84:1 (2008), 69-98.

В. П. Маслов

Московский государственный университет им. М. В. Ломоносова

E-mail:v.p.maslov@mail.ru 